\title{
BMJ Open Protocol for a scoping review on nursing care and the autonomy of disabled
} persons

\author{
Soraia Dornelles Schoeller, ${ }^{1}$ Daniella Karine Souza Lima, ${ }^{1}$ Maria Manuela Martins, ${ }^{2}$ \\ Flávia Regina Souza Ramos, ${ }^{3}$ Milena Amorim Zuchetto, ${ }^{1}$ \\ Luciana Neves da Silva Bampi, ${ }^{1}$ Cristine Moraes Roos, ${ }^{1}$ Adriana Dutra Tholl, ${ }^{1}$ \\ Liliam Cristiana Julio Tonnera, ${ }^{1}$ Wiliam Cesar Alves Machado, ${ }^{4}$ \\ Caroline Porcelis Vargas ${ }^{1}$
}

To cite: Schoeller SD, Lima DKS, Martins MM, et al. Protocol for a scoping review on nursing care and the autonomy of disabled persons. BMJ Open 2018;8:e22106. doi:10.1136/ bmjopen-2018-022106

- Prepublication history for this paper is available online. To view these files, please visit the journal online (http://dx.doi. org/10.1136/bmjopen-2018022106).

Received 1 February 2018

Revised 13 August 2018

Accepted 18 August 2018

Check for updates

(C) Author(s) (or their employer(s)) 2018. Re-use permitted under CC BY-NC. No commercial re-use. See rights and permissions. Published by BMJ.

${ }^{1}$ Rehabilitation Research Group, Nursing Department, Health Sciences Centre, Federal University of Santa Catarina, Florianopolis, Santa Catarina, Brazil

${ }^{2}$ Rehabilitation Research Group, Porto Nursing School, Porto, Portugal

${ }^{3}$ Nursing Department, Health Sciences Centre, Federal University of Santa Catarina, Florianopolis, Santa Catarina, Brazil

${ }^{4}$ Rehabilitation Research Group, Nursing Department, Health Sciences Centre, Federal

University of Rio de Janeiro, Rio de Janeiro, Brazil

\section{Correspondence to}

Dr Soraia Dornelles Schoeller; soraiadornelleschoeller@gmail. com

\section{ABSTRACT}

Introduction Considering the limitations that disabled persons experience, our understanding of caring for these people has evolved over time worldwide. Because nursing care is dependent on the choices made by patients, nurses can play an emancipatory role by empowering patients with disabilities to become the primary agent in their rehabilitation. Objective was to conduct a range analysis protocol proposing the mapping of conceptual elements for a nursing care model based on the autonomy of disabled persons.

Methods and analysis A protocol was developed in corporating insights from recent innovations and applying the scope review method of Arksey and 0'Malley as a reference using their scoping review methodology as a guide. For the construction of research guiding question, the patient, intervention, comparison and outcomes strategy was adapted. Considerable research in online databases (14) from 2000 to 2018 will be integrated by bibliographical research. Proceed a consult in the Annals of the Brazilian Congress of Nursing, Brazilian Nursing Association and Portuguese Rehabilitation Nursing Association, as well as professional Portuguese and Brazilian nursing legislation. The entire reference list of the covered studies searched manually to detect considerable additional studies. In addition to these searches, stakeholders, including nurse experts in the rehabilitation field and disabled persons, will be requested and included. Ethics and dissemination This study does not require ethical approval because it is a review and collection of data on publicly available materials. The results of this research will be published in a relevant journal on the subject of rehabilitation and presented at international scientific events in the area of rehabilitation nursing. Thus, the elaboration of this protocol further maps the gaps and strengthens the concepts capable of presenting strong evidence which assists and supports safer, smarter and more objective care for those living with permanent daily care obligations.

\section{BACKGROUND}

It is estimated that there are more than one billion people in the world with some kind
Strengths and limitations of this study

- This is a new treatment of a review to account for the large volume of literature on an extensive topic, enabling a research map on conceptual elements for a nursing care model based on the autonomy of disabled people.

- The search procedure includes 14 online peer-reviewed databases and a wide range of bibliographical research sources outside of these databases. Furthermore, we inform ourselves with the Annals of the Brazilian Nursing Congress, Brazilian Nursing Association and Portuguese Rehabilitation Nursing Association, as well as Portuguese and Brazilian professional nursing legislation.

- Stakeholders, including nurse experts in the rehabilitation field and disabled persons, during the review, will be requested and included.

- Scoping reviews inherently provide breadth and not depth about a certain topic; however, this study will provide a breadth of knowledge about a nursing care model based on the autonomy of people with physical disabilities.

of disability, of whom almost 200 million are experiencing considerable obstacles in their functioning. ${ }^{1}$ In Brazil, an estimated $14.5 \%$ of the population (24.6 million people) has some type of disability. Of this population, $7 \%$ of people have physical disabilities, including tetraplegia, paraplegia, hemiplegia, partial or complete amputations, walking difficulties and the inability to stand or walk. In addition, a significant number of this population is influenced by barriers to healthcare access. $^{2}$ By considering the limitations that disabled persons experience, as well as environmental structures and community attitudes, our understanding of caring for these people has evolved over time worldwide.

Within the field of nursing, there are intensive debates on the purpose of nurses' work 
and how this work is practised. One consensus in this debate is that nurses should be responsible for therapeutic care. The Brazilian guidelines assume that nurses identify the biopsychosocial dimensions that affect patients and promote the integral health of patients in every process. This requires that nurses use their own tools to enable this deep knowledge and comprehend personalised care. This also means nurses must respect patient autonomy, including respect for their choices and commitment to empower patients to make their own decisions. ${ }^{3}$

Autonomy is a complex and multifaceted term that has been the object of reflection and philosophical debate since ancient times. Immanuel Kant was one of the philosophers who most studied this subject. According to Kant, autonomy is associated with freedom, human rights, equality and dignity, among other values, in the sense that each person must be respected in relation to their choices and to the strategies that they define to achieve those choices. ${ }^{4}$

When providing nursing care of disabled persons, the principle of autonomy becomes even more indispensable. When patients are faced with relearning how to perform tasks that were easier before their disability, nurses can be instrumental in empowering patients in this learning process. In this manner, nursing care plays an emancipatory role by listening to the patient's choices and empowering them to become the primary agent of their rehabilitation. ${ }^{5}$

Autonomy is one of the purposes of rehabilitation. Only those who lose their skills know their limits and the possibilities they have to become skilful once again. In rehabilitation nursing, "we depend on will power, rebellion and persistence of our patient. ${ }^{5}$ Here, there is a double challenge regarding autonomy: 'the moment when this is the essence of the nursing work purpose and the interface of rehabilitation, in which, nothing can be achieved without the ill person really wanting to achieve. ${ }^{5}$

Considering the above, the elaboration of protocols is a relevant part of the design of scope evaluations, since it amplifies the transparency of the methods and authorises that the readers qualify their validity and trustworthiness, using appropriately. The objective was to conduct a range analysis protocol proposing the mapping of conceptual elements for a nursing care model based on the autonomy of disabled persons.

\section{METHODS}

Scope review is a perfect method for finding key concepts within a study area, detecting primary elements and categories of available samples, and identifying gaps in existing studies. Scope revisions and systematic reviews are not the same, the systematic ones aim to find answers to a specific research question, compiling the empirical evidence that fits into specific election criteria. ${ }^{6}$

This methodological study had as objective to present a protocol for a scope review of theoretical elements for the construction of a nursing care model based on the autonomy of people with physical disabilities. Its primary purpose is to assess the extent, breadth and depth of studies of a certain theme, as the first approximation of a specific topic considered or a very complex or littleknown topic.

Our protocol was developed applying the scope review method of Arksey and $\mathrm{O}^{\prime}$ Malley ${ }^{7}$ as a reference, as well the review of Levac $e t a l^{8}$ and further developed by the Joanna Briggs Institute. ${ }^{9}$ Six methodological stages are characterised: (1) identification of the research question; (2) identification of relevant studies (search for relevant studies); (3) selection of studies; (4) data extraction; (5) interpretation, summarisation and dissemination of the results and (6) (optional) consultation with stakeholders.

\section{Stage 1: identifying the research question}

The study starts with the elaboration of one research issue or more. The adaptation of the PICO technique was applied to elaborate the guiding question (P: patient; $\mathrm{I}$ : intervention; C: comparison; $\mathrm{O}$ : outcomes), with ' $\mathrm{P}$ ' representing the study population (people with physical disability), 'I', the phenomenon of interest (nursing care) and ' $\mathrm{C}$ ', the context (autonomy). In order to meet readers' needs, the PICO strategy can provide a great deal of information about the focus, scope and applicability of a review. For our study, a participatory process that included discussions on staff was used to develop the research question defined according to the PICO strategy, among others. ${ }^{10}$ The research questions produced were determined according to table 1 .

\section{Stage 2: identifying relevant studies}

The team discussions regulate the eligibility criteria, databases, descriptors, keywords and search strategies.

\section{Eligibility criteria}

Empirical and theoretical studies, published in English, Spanish or Portuguese, will be included between January 2000 and January 2018. The following types of documents in the selected databases will be included: original articles on qualitative (eg, randomised controlled trials, case-control studies, prospective or retrospective cohort studies, or quasi-experimental studies) and quantitative research, experience reports, literature reviews, integrative and systematic reviews with or without meta-analysis, scope reviews, guidelines, booklets, protocols, theses and dissertations. In addition, the reference lists of selected publications will be reviewed for any other relevant studies on the study topic.

\section{Databases}

The application of a search of articles published in online databases will allow the recognition of relevant studies, will be used as bases: Latin American and Caribbean Health Sciences; Spanish Bibliographic Index on Health Sciences; BDENF (Nursing Database); Cumulative Index to Nursing and Allied Health Literature; SCOPUS; Medical Literature Analysis and Retrieval System Online (PubMed/MEDLINE); Web of Science; Cochrane Library 
Table 1 Research questions developed by team discussions

\section{Research question (patient, intervention, comparison and outcomes strategy)}

What are the conceptual elements that constitute nursing care for the autonomy of people with physical disabilities?

\section{Other research questions}

What are health interventions for autonomy?

- How does the care relationship occur?

What is the responsibility of the person that is being cared for?

Which professionals are related to care?

- How is rehabilitation perceived by the professional?

- How is rehabilitation perceived by the patient?

- How does the professional feel about the work performed?

- What may be seen as weaknesses and potentialities for professional autonomy?

- What may be seen as weaknesses and potentialities for patient autonomy?

- How does the patient perceive the care relationship between themselves and the professional? and Scientific Electronic Library Online. In order to include all important information, several bibliographies of the grey type will be analysed, such as those found in Google Scholar, OpenGrey, PROQUEST, Capes Bank of dissertations and theses, and The Brazilian Ministry of Health.

Also will be consulted the Annals of the Brazilian Congress of Nursing, Brazilian Nursing Association and Portuguese Rehabilitation Nursing Association, as well as professional Portuguese and Brazilian nursing legislation. Beyond these organisations, the entire reference list of the covered studies will be searched manually to detect considerable additional studies.

\section{Search strategy}

PICO was used to elaborate the search method and considered the above-mentioned research questions. The literature search of articles was guided by Boolean operators AND and OR, as necessary. The database search technique was improved with the assistance of a library professional. We used Mendeley Desktop V.1.15.2. for the import of the references, and every search result was saved in documents.

This descriptors, keywords and their combinations were used to elaborate the strategy: 'Autonomy' OR 'Free Will' OR 'Self Determination' OR 'Freedom' AND 'Care' OR ‘Caring' AND 'Health' AND 'Disabled Person' OR 'Disabled Persons' OR 'Persons with Disabilities' OR 'Persons with Disability' OR 'Disabled persons' OR 'Physically Handicapped' OR 'Physically Disabled' OR 'Physically Challenged'.

\section{Stage 3: study selection}

The review method will be based on two degrees of choice: (1) a title and abstract review and (2) a full-text review. Initially, two independent researchers will make a choice of articles by reading the titles and abstracts of then, and this will be validated by a third researcher, thus choosing the appropriate articles. In a second moment, two researchers will independently select articles through all their content, determining their integration in the research. All full-text articles will be reread and re-evaluated when a disagreement occurs, and other disagreements about the choice of an article will be solved through discussion among researchers in search of agreement. In scope reviews, articles are not qualified by their methodological excellence, so it is not possible to eliminate articles that do not meet the methodological criteria sought.

Assistance will be requested from three experts on the subject of rehabilitation and disability for the selection and inclusion of studies in the list of references. They will receive the previously selected list and the instrument containing the research question and the search strategy used to facilitate the identification of relevant studies. The papers listed in the reference list will be included in the study. A Preferred Reporting Items for Systematic Reviews and Meta-Analyses flow scheme will be employed to enable the arrangement of the data.

\section{Stage 4: charting the data}

Two reviewers will obtain the data independently, which will be proven by a third person, and when there are uncertainties or inconsistencies, the authors will be asked to solve. The researchers will create an instrument to carry out the data collection, and it is possible to affirm the importance of each study and to draw from it its characteristics (type of publication, language, country and year), being these about the motive of the research, its proposal, researcher (name and place of work)and the article (journal, title, year and place of the research, methodology, sample, interventions, analysis, results and conclusions).

For each publication, the primary proposition of the problem, arguments, methods, discussions and conclusions will be identified and extracted. The primary focus will be analysed, turning to integral texts when necessary, from the categories of analysis identified that allow for the synthesis of the findings in a narrative form. The search indicated the path for the extraction and mapping of the findings. Bibliographies Management Software (Mendeley Desktop V.1.15.2) will assist with the 
organisation of the data. For stakeholder involvement (stage 6), the interview data will be collected either face to face or virtually (by Skype or email); it will be recorded and later transcribed.

\section{Stage 5: collating, summarising and reporting the results}

This scope study will detail the eminent topics that discuss the target population, areas of action, intervention characteristics and types of questions asked. This review research on the concept of nursing care for the autonomy of disabled persons will undercut potential gaps and will provide suggestions for future research.

The quantitative results will be presented by the absolute and relative frequencies of the studies found. Qualitative data from selected studies will be grouped into categories. The result of the interviews and stakeholder involvement (stage 6) will be analysed by Minayo et $a l^{11}$ from the exhaustive reading of interview transcripts to identify similarities and divergences of participant responses. Next, the data in the literature will be cross-checked with the data that emerged in the interviews. This iterative approach allows for the discussion of emerging issues and better exploitation of the data. The set of study data will present the results in the form of tables and flow charts.

\section{Stage 6: consultation with stakeholders}

The importance of the consultation stage by Arksey and $\mathrm{O}^{\prime}$ Malley ${ }^{7}$ and Levac et $a l^{8}$ is raised as a space for the understanding of stakeholders, sustaining insights ahead of what is found in the literature. Our research will incorporate the interests of stakeholders in two steps: (1) counselling with expert nurses in the rehabilitation field and disability, who will be in charge of listing aspects that are not yet known or that need further study and (2) consultations to get science over wisdom absences of research were conducted with patients or caregivers (table 2).

For data collection, the sample will be intentional. For the first stage, it will comprise 5 disabled persons living in Florianopolis-Brazil and 5 in the Porto-Portugal Region, totalling 10 disabled persons. For the second stage, the sample will comprise 5 nursing professionals living in
Florianopolis-Brazil and 5 in the Porto-Portugal Region, totalling 10 nursing professionals. The exclusion criterion for disabled persons in this study will be the impossibility of verbal communication (for this, the mini-mental questionnaire will be applied). At present, the exclusion criterion for nurses will be those who have not previously worked with disabled persons for at least 1 year. If five qualified nurses cannot be identified in Florianopolis-Brazil, other qualified nurses will be contacted from other locations via email, until the desired minimum number is reached.

\section{PATIENT INVOLVEMENT}

The contact will be made personally, either by phone or email, for disabled person and for nurses who get involved with the research. After being accepted to participate in the research and signing an informed consent form, the participants will be personally interviewed by the principal researcher according to his/her best interest by Skype or email.

This study will be conducted with nurse experts in the rehabilitation and disability field and disabled persons. Researchers will be committed for all phases of the study methodology, including interpretation of the results and editing, preparing and disseminating the manuscript for publication. All stakeholders address their valuable contributions through this study.

\section{DISSEMINATION AND ETHICS}

Since the transparency of research procedures has become a quality criterion for review, there is a growth in the publication of protocol restraints, making possible the publication in magazines of great impact. ${ }^{12}$ The results of this research will be published in a relevant journal on the subject of rehabilitation, which has peer review and qualifies nursing research and practice. All results of the review will also be announced at international scientific events in the area of rehabilitation

Table 2 Consultations with stakeholders composed of experts and patients

Consultant stakeholders Intervention proposal

Nurse experts in the rehabilitation and disability What does autonomy mean to you?

field

What kind of nursing care can help to achieve the autonomy of a person with a disability?

Report a situation in which you have the perception of what contributed to the autonomy of the disabled person.

How do you relate to autonomy, disability and nursing care? Is there anything else you want to add?

Disabled persons

Semistructured interview guide:

What does autonomy mean to you?

What kind of nursing care can help to achieve autonomy?

How can nurses help people with physical disabilities become autonomous?

Talk about a scenario where you felt autonomous after your disability.

Is there anything else you want to add? 
nursing. This research will be important for several audiences, covering researchers and health professionals. In particular, it will be important for nurses who provide care for disabled people. In these scenarios, the principles of autonomy and self-determination become necessary considerations, because disabled persons need to be rehabilitated to perform tasks that were once easy to perform before their disability. Also, the findings of this scope research will be useful for health organisations, universities and nursing professional leaderships (eg, the Brazilian Nursing Association and Portuguese Rehabilitation Association).

Recently, our research group conduced a protocol for a scoping review on skin self-care in people with spinal cord injury using 13 electronic databases from the last 10 years; this review is in the process of being finalised. ${ }^{13}$ Our research group is a pioneer in the rehabilitation field for disabled persons in Brazil. Therefore, the purpose of our studies is to first understand the state of the field and subsequently create evidence-based strategies regarding the complexity of rehabilitation care.

Our protocol was presented for the purpose of reviewing the scope of published material related to conceptual elements for a nursing model based on the autonomy of the person with disability in the past 18 years. This type of research that aims to condense a large amount of bibliographical research is modern, especially on the theme of nursing care strategies based on the autonomy of people with disabilities. This approach will also identify specific potential trends and gaps in this field, constituting the first step towards the future construction of a theoretical model of nursing care aimed at the autonomy of people with disability.

\section{CONCLUSIONS}

The care for disabled persons must continue throughout their life trajectory. The need of autonomy in this setting has strong impact on these peoples' rehabilitation and quality of life.

The development of conceptual elements for a nursing care model based on the autonomy of the disabled person collaborates and subsidises for a more protected, competent and skilled care for all who experience the inevitability of daily care dependency permanently. Moreover, because this topic is complex and still misunderstood, the elaboration of this protocol maps the gaps in the literature and strengthens concepts capable of improving research to guide future nursing care.
Contributors SDS and DKSL conceived and developed the research question and study methods and contributed meaningfully to the drafting and editing of the manuscript. SDS and DKSL also approved the final manuscript. MMM, FRSR, MAZ, LNdSB, CMR, ADT, LCJT, WCAM and CPV aided in developing the research question and study methods, contributed meaningfully to the drafting and editing of the manuscript and approved the final manuscript.

Funding The authors have not declared a specific grant for this research from any funding agency in the public, commercial or not-for-profit sectors.

Competing interests None declared.

Patient consent Not required.

Ethics approval The exploration of scope has as a method the review and collection of data only in materials that are released to the public, and therefore does not require ethical approval. However, the people participating in this study (stage 6) will be expected to follow ethical and legal guidelines, including Resolution no. 466/12 of the National Health Council and approval by the Human Research Ethics Committee of our Institutions via platform of No. C 1012154 dated 4 April 2015.

Provenance and peer review Not commissioned; externally peer reviewed.

Open access This is an open access article distributed in accordance with the Creative Commons Attribution Non Commercial (CC BY-NC 4.0) license, which permits others to distribute, remix, adapt, build upon this work non-commercially, and license their derivative works on different terms, provided the original work is properly cited, appropriate credit is given, any changes made indicated, and the use is non-commercial. See: http://creativecommons.org/licenses/by-nc/4.0/.

\section{REFERENCES}

1. The World Health Organization, 2011. World report on disability. accessed dez.2017 http://www.who.int/disabilities/world_report/ 2011/report.pdf.

2. Instituto brasileiro de geografia e estatística. Censo. 2000. acessed em 1 Feb 2018 http://www.ibge.gov.br.

3. Risjord M. Nursing and human freedom. Nurs Philos 2014;15:35-45.

4. Victor A, Hostmaelingen N. Direitos das Pessoas com Deficiência. Lisboa: Portugal, 2017.

5. Schoeller SD, Padilha M, Ramos FRS, et al, 2014. Pesquisa em enfermagem de reabilitação: apontamentos da realidade brasileira http://www.esenf.pt/fotos/editor2/i_d/publicacoes/978-898-98443-15.pdf (accessed Jan 2018).

6. Colquhoun HL, Jesus TS, O'Brien KK, et al. Study protocol for a scoping review on rehabilitation scoping reviews. Clin Rehabil 2017;31:1249-56.

7. Arksey H, O'Malley L. Scoping studies: towards a methodological framework. Int J Soc Res Methodol 2005;8:19-32.

8. Levac D, Colquhoun H, O'Brien KK. Scoping studies: advancing the methodology. Implement Sci 2010;5:69.

9. The Joanna Briggs Institute, 2015. Methodology for JBI Scoping Reviews Joanna Briggs Institute Reviewers' Manual: 2015 Edition http://joannabriggs.org/assets/docs/sumari/Reviewers-Manual Methodology-for-JBIScoping-Reviews_2015_v2.pdf (accessed 9 Feb 2017).

10. da Costa Santos CM, de Mattos Pimenta CA, Nobre MR. The PICO strategy for the research question construction and evidence search. Rev Lat Am Enfermagem 2007;15:508-11.

11. Minayo MCS, Souza ER, Paula DR. Revisão sistemática da produção acadêmica brasileira sobre causas externas e violências contra a pessoa idosa. Ciência \& SaúdeColetiva 2010;15:6:2709-18.

12. Luna Filho B, 1998. Basic stepsequence in the development ofresearch protocols. Arq Bras Cardiol;71:735-40 http://www.scielo. br/pdf/abc/v71n6/a01v71n6.pdf.

13. Lima DKS, Schoeller SD, Knihs NDS, et al. Protocol for a scoping review of skin self-care of people with spinal cord injury. BMJ Open 2017;7:9. 